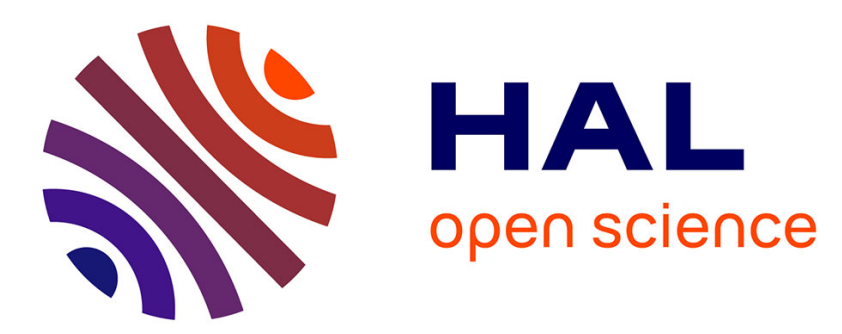

\title{
Balance magnétique appliquée à l'étude des phénomènes de précipitation
}

\author{
A. Junqua, J. Mimault, J. Delafond
}

\section{To cite this version:}

A. Junqua, J. Mimault, J. Delafond. Balance magnétique appliquée à l'étude des phénomènes de précipitation. Revue de Physique Appliquée, 1972, 7 (4), pp.373-378. 10.1051/rphysap:0197200704037300 . jpa-00243641

\section{HAL Id: jpa-00243641 https://hal.science/jpa-00243641}

Submitted on 1 Jan 1972

HAL is a multi-disciplinary open access archive for the deposit and dissemination of scientific research documents, whether they are published or not. The documents may come from teaching and research institutions in France or abroad, or from public or private research centers.
L'archive ouverte pluridisciplinaire HAL, est destinée au dépôt et à la diffusion de documents scientifiques de niveau recherche, publiés ou non, émanant des établissements d'enseignement et de recherche français ou étrangers, des laboratoires publics ou privés. 
Classification

Physics Abstracts

17.60

\title{
BALANCE MAGNÉTIQUE APPLIQUÉE A L'ÉTUDE DES PHÉNOMẼNES DE PRÉCIPITATION
}

\author{
A. JUNQUA, J. MIMAULT et J. DELAFOND
}

Laboratoire de Métallurgie Physique associé au CNRS (L. A. 131). UER Sciences Fondamentale et Appliquée

(Reçu le 7 janvier 1972, révisé le 14 juin 1972)

\begin{abstract}
Résumé. - Nous décrivons une balance magnétique adaptée à l'étude des phénomènes de précipitation dans les alliages à faible susceptibilité tels que les alliages d'aluminium avec des métaux " normaux ». Les mesures peuvent être réalisées sous une gamme étendue de températures allant de $15^{\circ} \mathrm{K}$ à $900^{\circ} \mathrm{K}$. La sensibilité du dispositif est de $10^{-10}$ uem CGS/g.

Nous donnons dans la deuxième partie quelques résultats relatifs à l'aluminium et aux alliages $\mathrm{Al}-\mathrm{Cu}$.
\end{abstract}

Abstract. - A magnetic balance for the study of precipitation in weak susceptibility alloys, like aluminium alloyed with « normal » metals, is described ; this device can be used for measurements between $15^{\circ} \mathrm{K}$ and $900^{\circ} \mathrm{K}$ with a sensibility of $10^{-10}$ uem CGS/g.

In the second part of this article, we present some results obtained with aluminium and $\mathrm{Al}-\mathrm{Cu}$ alloys.

Quelques auteurs (Sleppy [1], H. Borchers et W. Hepp [3]) ont utilisé la susceptibilité statique comme paramètre physique d'étude de la préprécipitation dans les alliages d'aluminium. Nous avons constitué un montage qui par sa sensibilité et sa facilité d'utilisation permet l'étude de ces phénomènes. Nous décrirons en détail la réalisation de ce montage, avec les considérations techniques qui nous ont amenés à choisir chacun des éléments. A titre d'exemple, nous donnerons les résultats obtenus pour deux échantillons d'aluminium d'origines, donc de puretés différentes et aussi pour différents alliages aluminium-cuivre avec des concentrations en $\mathrm{Cu}$ allant jusqu'à $4 \%$ en poids.

Nous nous proposons d'étudier avec une précision suffisante les variations de susceptibilités dues à la précipitation dans des métaux normaux tels que l'aluminium. La susceptibilité de l'aluminium à la température ambiante est d'environ $0,61 \times 10^{-6}$ uem CGS/g. Les variations attendues sont de l'ordre du centième de cette valeur; afin d'exploiter au mieux les résultats il nous a semblé nécessaire de nous fixer une précision de $1 \%$ sur ces variations. De ce fait, la sensibilité globale recherchée devra être de $10^{-10}$ uem $\mathrm{CGS} / \mathrm{g}$.

I. Description de l'appareillage. - Nous avons adopté la méthode de Faraday parce qu'elle permet d'opérer sur des échantillons de petites dimensions ; c'est seulement dans ces conditions que l'on peut obtenir des traitements thermiques homogènes.

a) Aimant et alimentation. - Pour évaluer la valeur de $B \cdot \frac{\partial B}{\partial x}$ nécessaire nous écrivons qu'une variation de susceptibilité de $10^{-10}$ CGS entraine une variation de force de $0,1 \mu \mathrm{g}$. Pour cela :

$$
B \cdot \frac{\partial B}{\partial x}=3 \times 10^{6} \text { gauss }^{2} / \mathrm{cm} \text {. }
$$

Nous avons choisi un aimant Spectromagnétic permettant un produit $B \cdot \frac{\partial B}{\partial x}$ égal à $10^{7}$ gauss $^{2} / \mathrm{cm}$ et obtenu pour un entrefer de $38 \mathrm{~mm}$ "et une intensité de $95 \mathrm{~A}$. L'axe des $x$ est l'axe vertical passant par le centre de l'échantillon. Pour un échantillon d'aluminium de $5 \mathrm{~mm}$ de hauteur, nous avons pu vérifier qu'un déplacement de $1 \mathrm{~mm}$ selon l'axe des $x$ autour de la position correspondant au maximum de force n'engendrait aucune variation de force qui puisse être détectée, le dispositif travaillant à sa plus grande sensibilité $(0,1 \mu \mathrm{g})$.

Les mesures de susceptibilité magnétique par une méthode statique peuvent être perturbées par des contaminations ferromagnétiques d'autant plus gênantes que la susceptibilité est faible. Dans notre cas particulier de mesures sur des alliages à base d'aluminium, nous avons pris beaucoup de précautions dans la manipulation de nos échantillons pour éviter toute contamination extérieure ; de plus, le faible taux d'impuretés décelé par les analyses (nous présentons les résultats de ces analyses par ailleurs) nous permet de penser que les traces d'éléments ferromagnétiques contenues dans les échantillons ne modifient pas la valeur de leur susceptibilité puisque ce sont des alliages à base d'aluminium.

Nous avons travaillé avec un champ moyen de l'ordre de $10^{4}$ gauss.

- Il faut d'autre part que les fluctuations d'intensité ne produisent que des variations de force très inférieures à $0,1 \mu \mathrm{g}$. 
Mais $F=K I^{2}$ et $\Delta F / F=2 \Delta I / I$ pour l'aluminium $F \sim 2000 \mu \mathrm{g}, \Delta I / I \sim 5 \times 10^{-5}$.

Nous avons choisi une alimentation du type résonance électronique régulée en courant à $10^{-6}$. Ceci nous permet de suivre des évolutions de faible amplitude sur des temps de l'ordre de la journée.

b) Structure D'ENSEMble DE L'APPAREILlage (Fig. 1). - La mesure de force est faite à l'aide d'une

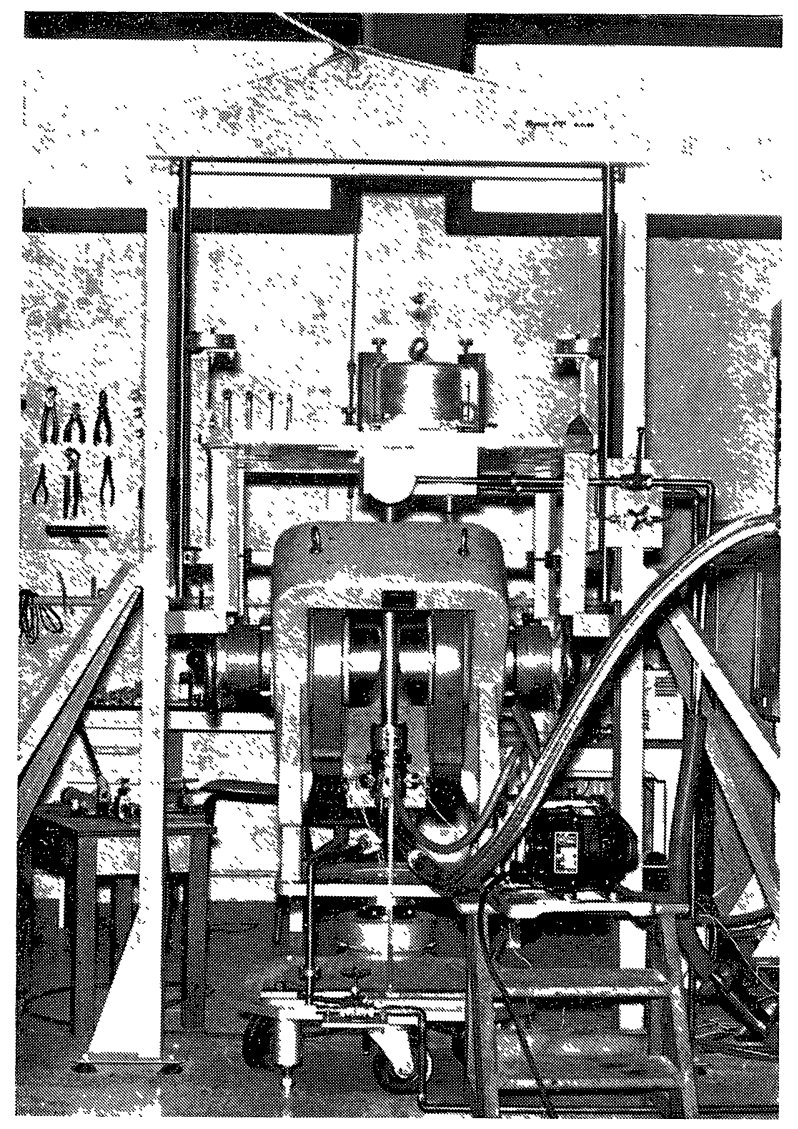

Fig. 1. - Photographie du montage dans le cas des mesures à à basses températures.

balance Cahn : sensibilité $0,1 \mu \mathrm{g}$, portée $1 \mathrm{~g}$. Un vérin hydraulique permet de placer l'échantillon facilement et d'une manière reproductible dans la zone de force maximale.

Nous avons étudié un bâti permettant le dégagement complet de la balance et son retour en position de mesure, strictement guidé par un ensemble de huit roulements à bille s'appuyant sur un chemin de roulement en acier rectifié. Cette disposition permet de changer rapidement d'échantillon.

Un groupe de pompage (pompe à palettes + pompe à diffusion) permet d'obtenir un vide secondaire dans toute l'enceinte de mesure.

Pour obtenir les basses températures, nous utilisons l'hélium comme gaz d'échange sous une pression de $2 \mathrm{~cm}$ de $\mathrm{Hg}$. Un système distributeur comportant une vanne pointeau permet d'ajuster cette pression. c) RÉGlage et Mesure des températures. - Un four Setaram (Fig. 2) nous permet d'effectuer des mesures à toutes températures comprises entre l'am-

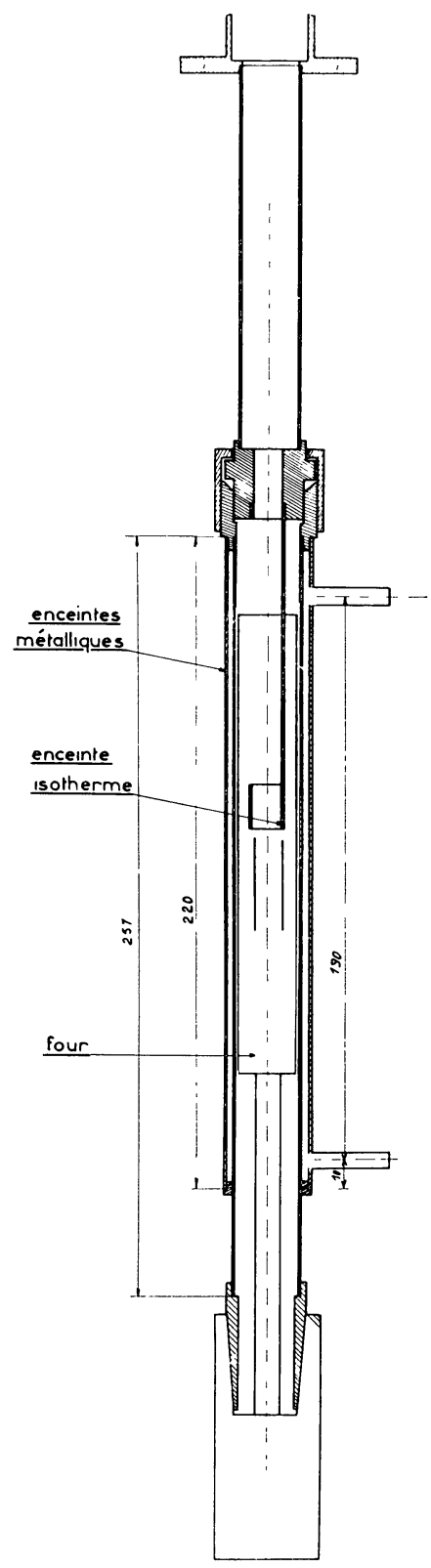

FIG. 2. - Schéma du four.

biante et $600^{\circ} \mathrm{C}$. Ainsi nous avons procédé «in situ » aux opérations de revenu et nous pouvons suivre les cinétiques de précipitation sans manipuler l'échantillon. Un régulateur statique (CORECI) utilisant un thermocouple comme détecteur donne une précision de régulation de $1 / 2^{\circ} \mathrm{C}$.

Le cryostat est du type à circulation (Air-Liquide) et permet de travailler de façon permanente à toute température comprise entre $15^{\circ} \mathrm{K}$ et l'ambiante. La régulation (MERIC) utilisant une sonde As-Ga comme détecteur donne une précision de régulation de l'ordre de $4 / 100^{\circ} \mathrm{C}$. 
La précision recherchée dans les mesures de forces nous interdit toutes les mesures où l'échantillon est en contact mécanique avec le détecteur. Nous avons placé le thermocouple $\mathrm{Pt}-\mathrm{Pt}$. Rhodié le plus près possible de l'échantillon dans la zone homogène du four, ce qui nous permet de connaître les températures avec une précision de l'ordre de $1^{\circ} \mathrm{C}$. Dans le domaine des basses températures, la précision doit être meilleure. Les mesures se font à l'aide d'une sonde As-Ga placée dans le corps dú cryostat. Pour une pression de gaz d'échange donnée, nous avons pu établir une correspondance entre la température de l'échantillon et celle donnée par la sonde. Pour ceci, dans la gamme de températures comprises entre l'azote liquide et l'ambiante nous avons mesuré la susceptibilité du $\mathrm{CuSO}_{4}, 5 \mathrm{H}_{2} \mathrm{O}$, ce corps obéissant à la loi de Curie. Cette mesure permet donc de connaître la température de l'échantillon, par conséquent d'étalonner la sonde As-Ga. Pour des températures comprises entre l'hélium et l'azote liquide, nous avons employé le nitrate de Cérium-Magnésium et procédé de la même façon. d) Présentation des échantillons. - Dans le cas de mesures absolues la forme de l'échantillon est importante pour effectuer la correction due au champ démagnétisant. Dans notre cas nous n'effectuerons que des mesures relatives; cette correction n'intervient pas si nous donnons la même forme à l'échantillon de référence et à ceux qui sont à étudier. L'échantillon a la forme d'un cylindre de $6 \mathrm{~mm}$ de diamètre et $5 \mathrm{~mm}$ de hauteur, surmonté d'une tête cylindrique $(2 \mathrm{~mm}$ de diamètre et $2 \mathrm{~mm}$ de hauteur) elle-même percée d'un trou afin d'accrocher l'échantillon à la boucle inférieure du fil.

A haute température le frottement des molécules gazeuses provoque la création de charges électrostatiques. Elles n'ont pu être éliminées correctement que par l'emploi d'enceintes métalliques reliées à la masse. Refroidies par une circulation d'eau, ces enceintes ont un autre avantage important qui est le blindage contre les rayonnements électromagnétiques (schéma Fig. 2).

\section{Composition des échantillons :}

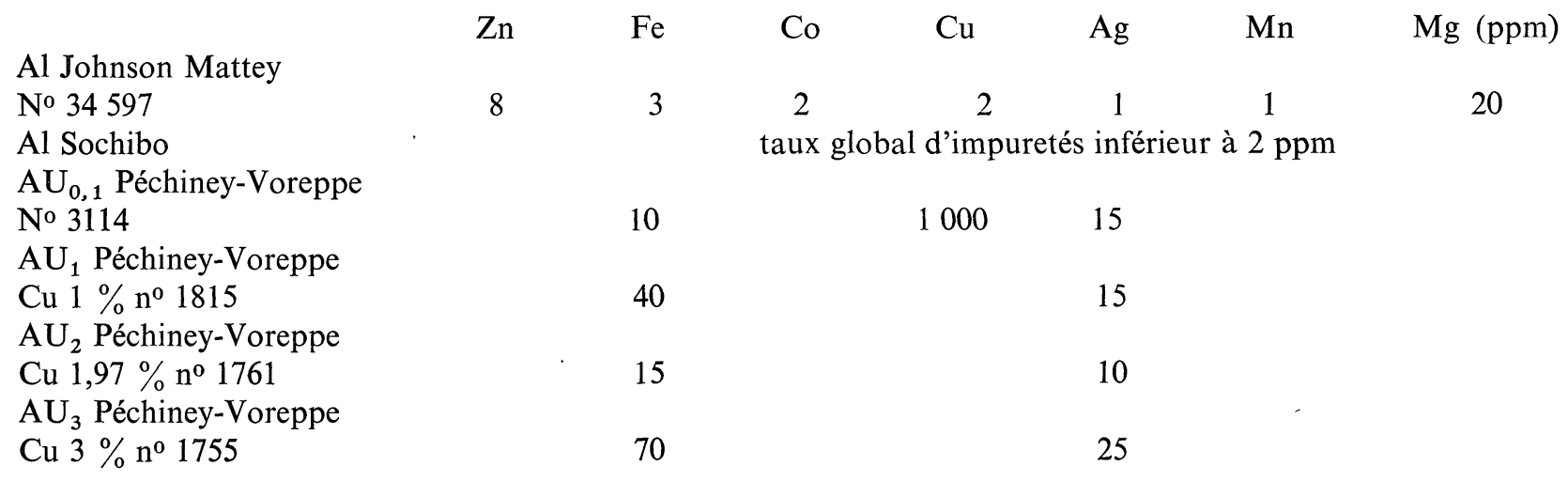

Les mesures à haute température peuvent être faites sous des pressions pouvant aller jusqu'à la pression atmosphérique. Or, un échantillon placé sous atmosphère gazeuse ou sous vide, est soumis, s'il est chauffé dans un four, à des forces parasites verticales de l'ordre du milligramme dans certaines conditions ; en effet, un gradient de température engendre des courants de convection. Pour supprimer cet inconvénient, il suffit de placer l'échantillon dans une enceinte « isotherme » [[10], [11] et schéma (Fig. 2)] construite en acier inoxydable amagnétique, cette enceinte cylindrique est fermée en bas et ouverte en haut pour laisser passer l'échantillon qui, lui, est suspendu à l'extrémité d'un fil d'or de $1 / 10 \mathrm{~mm}$ de diamètre ; l'or a été choisi pour sa faible susceptibilité indépendante de la température. La force magnétique calculéé s'exerçant sur le fil est de l'ordre de $30 \mu \mathrm{g}$, par conséquent ses variations avec la température seront absolument négligeables. Comme d'autre part nous faisons des mesures relatives de susceptibilité, sa grandeur elle-même n'intervient pas.

Les variations de la longueur du fil d'or avec la température pourraient être aussi un inconvénient ; si on considère qu'une partie seulement du fil $(10 \mathrm{~cm}$ environ) subit l'influence thermique, la variation de longueur n'excède pas $1 \mathrm{~mm}$ p̋our une élévation de température de $1000{ }^{\circ} \mathrm{C}$; de ce fait, la perturbation apportée à la mesure de la force est inférieure à $0,1 \mu \mathrm{g}$.

Nous avons constaté qu'un vide primaire suivi d'une mise sous pression réduite d'He laisse un taux de gaz résiduels beaucoup trop élevé et que la condensation de ces gaz sur l'échantillon au cours du refroidissement provoque des dérives importantes. Un calcul approché nous rend compte de ces résultats. Prenons un gaz de poids atomique moyen, l'air par exemple ; il reste dans l'enceinte $10^{-3} \mathrm{~g}$ d'air, si l'on considère que cet air se condense également sur les parois du cryostat et sur l'échantillon; le rapport des surfaces étant d'environ $2 / 65$ on constate qu'il se dépose $35 \mu \mathrm{g}$ d'air sur l'échantillon, ceci est tout à fait incompatible avec la précision recherchée. C'est pourquoi nous avons monté un groupe de pompage secondaire permettant d'atteindre des vides meilleurs que $10^{-4} \mathrm{~mm}$ $\mathrm{Hg}$.

D'autre part, à la remontée en température un phénomène de bombardement de l'échantillon par des 
molécules qui s'évaporent produirait également des forces parasites importantes.

Le dispositif ainsi mis au point nous permet de détecter des changements de susceptibilité de $1 \times 10^{-10}$ uem/ CGS sur les alliages d'Al étudiés.

II. Un exemple d'application : étude de l'Al et de l'Al-Cu. - Dans ce paragraphe, nous donnerons essentiellement les résultats de mesure pour montrer les possibilités de l'appareillage. L'exploitation détaillée de ces résultats fera l'objet d'autres publications.

1) $\mathrm{Al}$ (Johnson-MATteY), $\mathrm{Al}$ (SochiBo), $\mathrm{Al}-\mathrm{Cu}$ $0,1 \%$ - Préparation des échantillons. - Après usinage les échantillons sont polis électrolytiquement avec le bain alcool éthylique-acide perchlorique, ensuite nous leur faisons subir un traitement de revenu afin d'éliminer les effets d'écrouissage apportés par l'usinage, ils sont ensuite repolis et amenés à un poids d'environ $300 \mathrm{mg}$.

Les résultats obtenus sont montrés figure 3. Nous avons effectué la correction due à la dilatation du réseau.

$$
\frac{\Delta \chi}{\chi}=\frac{2}{3} \frac{\Delta V}{V}=2 \alpha T
$$

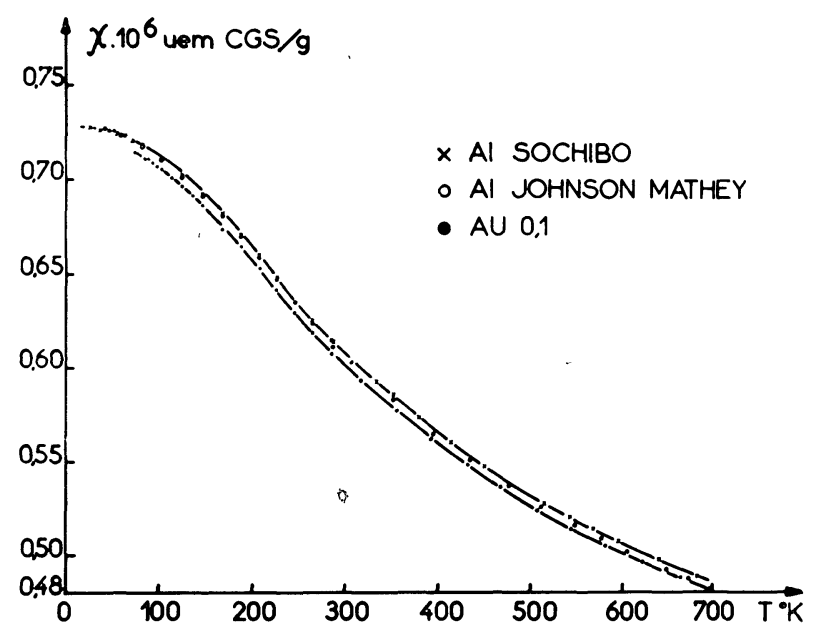

FIG. 3. - Susceptibilité magnétique d'Al de pureté différente en fonction de la température.

$\alpha$ : coefficient de dilatation linéaire supposé constant dans le domaine de température exploré,

$T$ : température absolue.

La théorie prévoit que la variation de susceptibilité en fonction de la température est de la forme, dans le cas où $k T \ll E_{\mathrm{F}}$ :

$$
\chi(T)=A-B T^{2}
$$

$k$ : constante de Boltzmann,

$E_{\mathrm{F}}$ : énergie du niveau de Fermi.

Certains auteurs [2], [4] ont trouvé des courbes expérimentales vérifiant cette loi dans un domaine restreint de température. La meilleure précision de nos mesures et une gamme plus étendue montrent que cette loi n'est qu'une première approximation (Fig. 4). D'autre part, on remarque que la forme des courbes dépend peu de la quantité d'impuretés. Cette allure

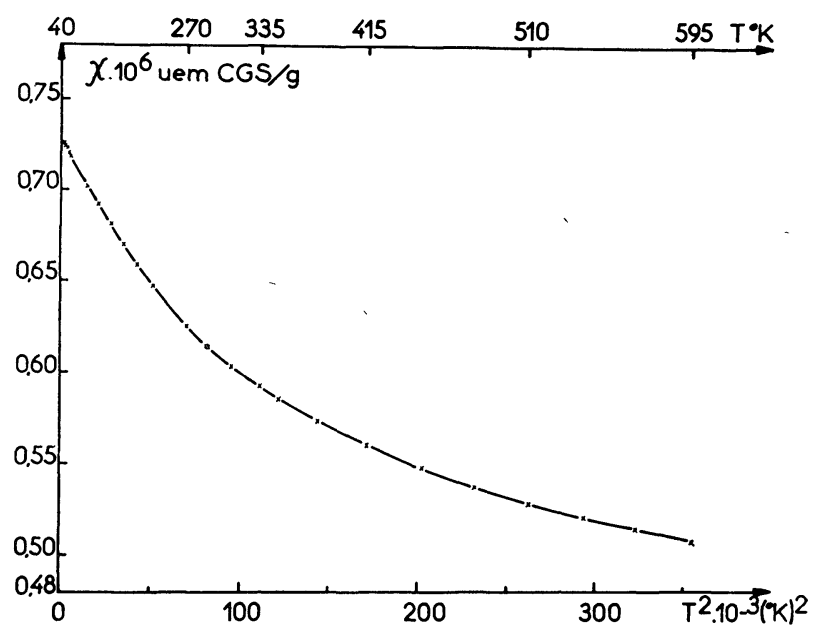

Fig. 4. - Susceptibilité de l'Al en fonction de $T^{2}$.

semble donc une propriété intrinsèque de l'Al. Cette indépendance relative par rapport aux impuretés est en accord avec les résultats de Hedgok et $\mathrm{Li}$ [2] et de J. C. G. Wheeler [4] sur la susceptibilité des alliages Al-Mn.

2) VieILliSSEMENT APRÈS TREMPE D'UN ALLIAGE $\mathrm{Al}-\mathrm{Cu} 4 \%$ (Fig. 5). - Le montage que nous avons

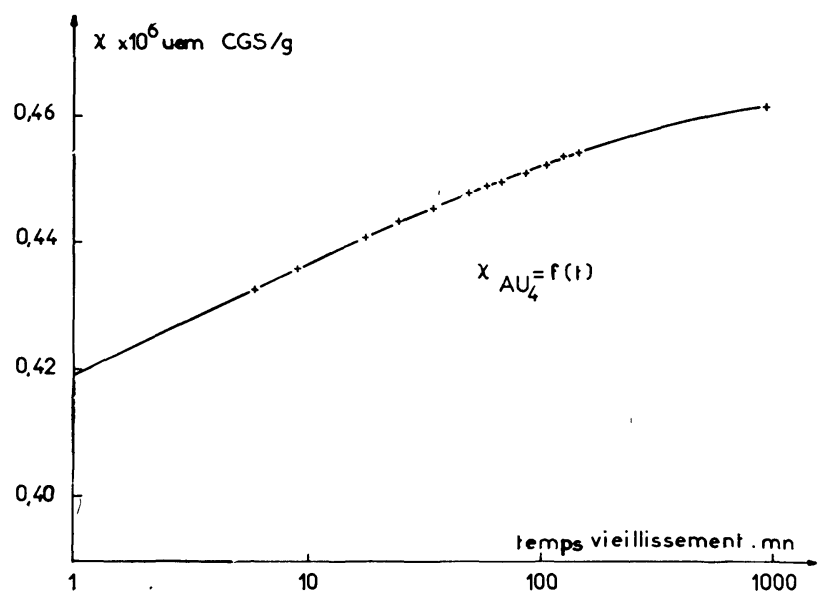

FIG. 5. - Cinétique de vieillissement de $\mathrm{AU}_{4}$ à $70{ }^{\circ} \mathrm{C}$.

réalisé nous permet de suivre la susceptibilité, d'une manière continue, au cours du temps, et à température constante. Ceci est particulièrement intéressant dans l'étude des phénomènes de précipitation. Nous donnons ici l'exemple d'un échantillon d'Al-Cu $4 \%$, ayant été précédemment trempé à l'eau à partir d'une température de $520^{\circ} \mathrm{C}$. Le résultat obtenu est comparable à ceux de Sleppy [1] et H. Borchers et W. Hepp [3].

3) EXPÉRIENCE DE TREMPES LENTES (Fig. 6). - Il est intéressant de connaître le comportement magnétique 


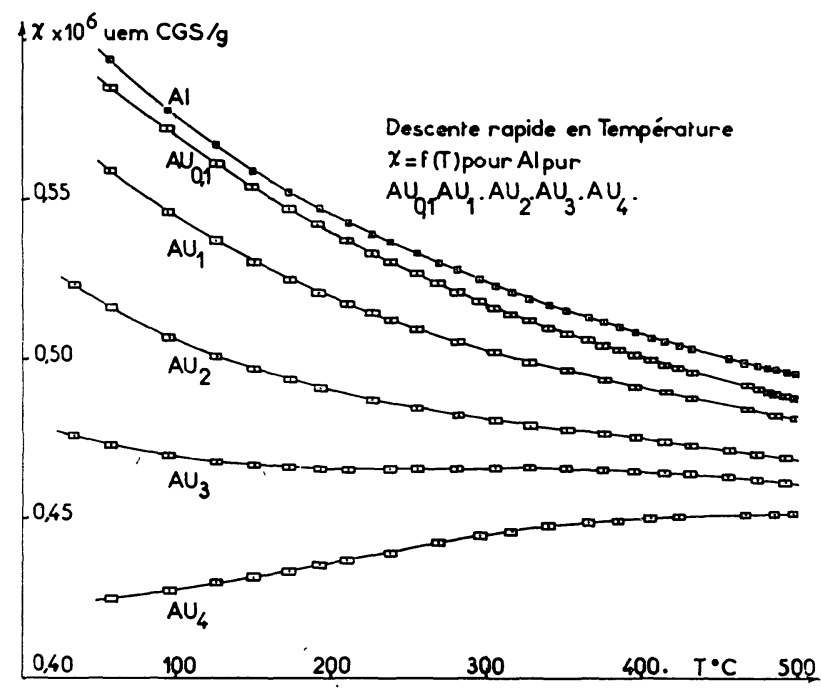

FIG. 6. - Après un recuit d'homogénéisation des échantillons à $520^{\circ} \mathrm{C}$ (ce recuit est fait en position de mesure dans l'entrefer), on coupe l'alimentation du four et on enregistre la susceptibilité en fonction de la température au cours du refroidissement.

d'un alliage au cours du refroidissement. Notre dispositif expérimental nous permet d'obtenir un refroidissement de $520^{\circ} \mathrm{C}$ jusqu'à l'ambiante en $8 \mathrm{mn}$, simplement en coupant l'alimentation du four. La température est évaluée en mesurant la susceptibilité d'un échantillon d'Al pur traité dans les mêmes conditions. Il est certain qu'une trempe à une vitesse aussi faible ne permet pas de conserver une sursaturation en lacunes, mais deux arguments nous font espérer la conservation d'un état voisin de l'état d'équilibre à haute température (le cuivre restant dispersé dans la matrice).

$1^{0}$ Après ce traitement thermique, nous retrouvons une valeur de susceptibilité voisine de celle extrapolée après trempe à l'eau.

$2^{\circ} \mathrm{Au}$ cours de cette descente rapide en température nous obtenons une loi de variation qui est superposable à celle obtenue après une expérience de réversion [5] ; or, il est admis qu'après ce traitement, les zones sont dissoutes et nous retrouvons la solution solide initiale.

Le réseau de courbes ainsi obtenu pour différentes concentrations de $\mathrm{Cu}$ nous permet de tracer la variation de susceptibilité avec la concentration pour toutes les températures intermédiaires. Nous constatons que, en première approximation, $\Delta \chi=\chi_{\mathrm{Al}}-\chi_{\text {Alliage }}$ (Fig. 7) est proportionnel à la concentration en poids de $\mathrm{Cu}$. D'autre part, nous avons noté que les courbes sont semblables à celles obtenues pour $\mathrm{Al}-\mathrm{Zn}$ par $\mathrm{H}$. Borchers et W. Hepp [3].

Les mesures de susceptibilité magnétique statique ne permettent pas de séparer les différentes contributions à la valeur de la susceptibilité

$$
\chi=\chi_{\text {ions }}+\chi_{\text {Pauli }}+\chi_{\text {Landau }} \text {. }
$$

H. J. Blythe et ses collaborateurs [6] ont montré en évaluant le paramagnétisme de Pauli par des mesures

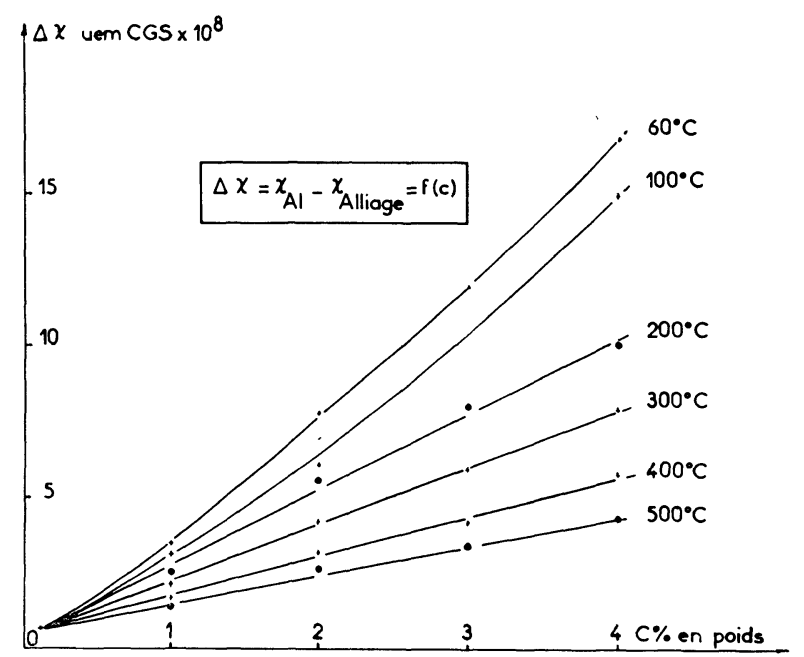

Fig. 7. - Ces courbes représentent l'écart qui existe entre les susceptibilités de l'aluminium et des alliages $\mathrm{Al}-\mathrm{Cu}$ en fonction de la concentration; cet écart qui a été déduit du réseau de courbes de la figure 6 est présenté pour différentes températures.

de chaleur spécifique à basses températures que c'est le diamagnétisme de Landau qui évolue dans de grandes proportions. La théorie la plus récente sur ce sujet, due à R. B. Thomas [7] donne dans le cas d'un alliage $\mathrm{Al}-\mathrm{Cu} 4 \%$ dans l'état où le cuivre est dispersé d'une manière aléatoire dans la matrice

$$
\Delta \chi=\chi_{\mathrm{A} 1}-\chi_{\text {Alliage }}=2 \times 10^{-11} \mathrm{CGS} / \mathrm{g}
$$

c'est-à-dire une contribution non visible expérimentalement. Cette théorie est donc insuffisante pour expliquer les effets constatés.

Conclusion. - Nous avons réalisé un montage expérimental nous permettant d'atteindre une précision suffisante pour pouvoir étudier les phénomènes de préprécipitation dans les alliages à base de métaux de très faible susceptibilité. Nous présentons les résultats expérimentaux sur l'alliage $\mathrm{Al}-\mathrm{Cu}$ comme exemple de mesure. Ce montage nous permet d'étudier les cinétiques de précipitation.

La précision des mesures effectuées sur l'Al montre que la variation de la susceptibilité en fonction de la température ne suit pas une loi $\chi=A-B T^{2}$ comme le prévoit la théorie. D'autres auteurs, par exemple J. C. Wheeler [4] employant la même méthode, ont trouvé des formes $\chi(T)$ et des valeurs analogues :

$$
\frac{\chi 80{ }^{\circ} \mathrm{K}-\chi 300{ }^{\circ} \mathrm{K}}{\chi 300^{\circ} \mathrm{K}}=18 \% \text {. }
$$

Cette valeur est en désaccord avec les résultats obtenus par des mesures de déplacement de Knight $K(T)$ [9] dans les mêmes conditions de température

$$
\frac{\Delta K}{K} \sim 2 \% \text {. }
$$

Il faut cependant remarquer que les mesures de 
déplacement de Knight se font à champ magnétique constant et sur des métaux en poudre.

La différence de susceptibilité entre l'Al et ses alliages est beaucoup plus importante que celle prévue par la théorie. D'autre part, la variation de la susceptibilité de ces alliages en fonction de la température est très différente de celle de l'Al pur et dépend de leurs états métallurgiques.

\section{Bibliographie}

[1] SLEPPY (W. C.). - Transactions of Metallurgical Society of AIME, 1967, 684, 239.

[2] Hedgcock (F. T.) and Li (P. L.). - Phys. Rev. B, 1970, 2.

[3] Borchers (H.) and HePp (W.). - Z Metallkde, 1969, 722.

[4] Wheeler (J. C. G.). - Phys. lett., 1968, 28A.

[5] Delafond (J.), Junqua (A.), Mimault (J.), Herve (J.). - Phys. lett., 1970, 32A.
[6] Blythe (H. J.), Holden (T. M.), Diwon (M.), Hoare (F. E). - Phil. Mag., 1965, 11, 235.

[7] Thomas (R. B. Jr.). - Phys. Rev., 1969, 185, 823.

[8] WiLson (A. H.). - The theory of Metals (Cambridge University Press), 1965, 168.

[9] Toshimoto, Kushida and Murphy (J. C.). - Phys. Rev. B, 1971, 3. 1574.

[10] Eyraud (Ch.) et Goton. - Bull. Soc. Chim., 1953, 20, 1009.

[11] Corbet (F.). - Thèse Lyon, 1960, 15-17. 